Title page

\title{
How does the workload and work activities of procedural GPs compare to non-procedural GPs?
}

\section{AUTHORS and AFFILIATIONS:}

Deborah J Russell, MBBS, FRACGP, MClinEpid, PhD, Research Fellow ${ }^{1,2,4,5}$

Matthew R McGrail, BSc(Hons), GradDiplT, PhD, Senior Research Fellow ${ }^{2,3,4}$

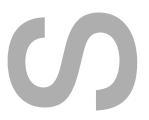

1 Monash Rural Health, Monash University, PO Box 666 Bendigo 3552, VIC

2 Centre of Research Excellence (CRE) in Rural and Remote Primary Health Care, PO Box 666 Bendigo 3552, VIC

3 Monash Rural Health, Monash University, Northways Road, Churchill, 3842, VIC

4 Centre of Research Excellence (CRE) in Medical Workforce Dynamics, http://mabel.org.au/

5 Corresponding author

Email: $\quad$ deborah.russell@monash.edu

Telephone: $\quad$ +61354409046

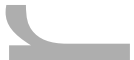

$+61400162821$

AUTHOR CONTRIBUTIONS:

\begin{tabular}{|l|c|c|}
\hline & Deborah Russell & Matthew McGrail \\
\hline CONTRIBUTION to the & $70 \%$ & $30 \%$ \\
\hline
\end{tabular}

This is the author manuscript accepted for publication and has undergone full peer review but has not been through the copyediting, typesetting, pagination and proofreading process, which may lead to differences between this version and the Version of Record. Please cite this article as doi: 10.1111/ajr.12321

This article is protected by copyright. All rights reserved 
Both authors made substantial contributions to the conception of this paper and to drafting the paper. Both authors agree to be accountable for all aspects of the work in this paper and have given their approval for submission of the paper in its current state.

\section{DISCLOSURE STATEMENT:}

This research has not been previously published in part or full elsewhere.

\section{ACKNOWLEDEGEMENTS:}

This work is part of the MABEL longitudinal study of Australian doctors. MABEL is funded by the National Health and Medical Research Council (Health Services Research Grant: 2008e2011; and Centre for Research Excellence in Medical Workforce Dynamics: 2012e2016), with additional funding from the Commonwealth Department of Health (in 2008) and Health Workforce Australia (in 2013). The views in this paper are those of the authors. We thank the other members of the MABEL team for their support and input, and especially acknowledge the doctors who give their valuable time to participate in MABEL.

\section{CONFLICT OF INTEREST:}

The authors are not aware of any conflict of interest being present.

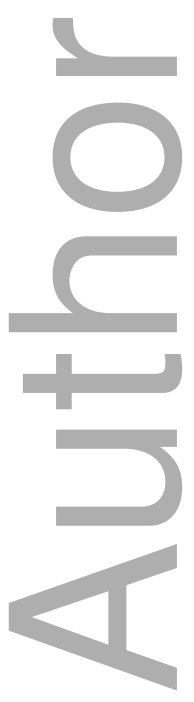

This article is protected by copyright. All rights reserved 


\section{How does the workload and work activities of procedural GPs compare to non-procedural GPs?}

Objectives: To investigate patterns of Australian GP procedural activity and associations with:

i. geographical remoteness and population size;

ii. hours worked in hospitals and in total; and

iii. availability for on-call

Design and participants: National annual panel survey (Medicine in Australia: Balancing Employment and Life) of Australian GPs, 2011-2013

Main outcome measures: Self-reported geographical work location, hours worked in different settings, and on-call availability per usual week, were analysed against GP procedural activity in anaesthetics, obstetrics, surgery or emergency medicine

Results: Analysis of 9301 survey responses from 4638 individual GPs revealed significantly increased odds of GP procedural activity in anaesthetics, obstetrics or emergency medicine as geographical remoteness increased and community population size decreased, albeit with plateauing of the effect-size from medium-sized (population 5000-15000) rural communities. After adjusting for confounders, procedural GPs work more hospital and more total hours each week than nonprocedural GPs. In 2011 this equated to GPs practising anaesthetics, obstetrics, surgery, and emergency medicine providing $8 \%(95 \% \mathrm{Cl} 0,16), 13 \%(95 \% \mathrm{Cl} 8,19), 8 \%(95 \% \mathrm{Cl} 2,15)$ and $18 \%$ $(95 \% \mathrm{Cl} 13,23)$ more total hours each week, respectively. The extra hours are attributable to longer hours worked in hospital settings, with no reduction of private consultation hours. Procedural GPs also carry a significantly higher burden of on-call. 
Conclusions: The longer working hours and higher on-call demands experienced by rural and remote procedural GPs demand improved solutions, such as changes to service delivery models, so that long-term procedural GP careers are increasingly attractive to current and aspiring rural GPs.

\section{Keywords \\ Doctors \\ Rural

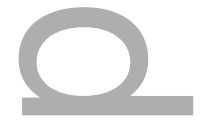 \\ Remote \\ Australia \\ Generalist}

\section{What is already known?}

- The services provided by procedural GPs enable rural and remote people to receive timely and potentially life-saving locally accessible health care

- The proportion of GPs who provide procedural services is declining

- Recently qualified GPs choosing rural practice are often interested in developing and maintaining procedural skills, but are simultaneously seeking a satisfactory work-life balance

\section{What does this paper add?}

- This paper demonstrates significant and strong associations between practising GP anaesthetics, obstetrics or emergency medicine and geographical remoteness/town population size

- Significant associations are also demonstrated between GP procedural activity and total hours worked each week, after adjusting for potentially confounding variables such as geographical location

- It is estimated that procedural GPs work approximately $8-18 \%$ more total hours each week compared to non-procedural GPs

\section{Introduction}

General Practitioners (GPs) practising anaesthetics, surgery, obstetrics and/or emergency medicine hereafter termed 'procedural GPs' - to rural and remote communities deliver important, if not essential, locally accessible medical care that is potentially life-saving. As geographical remoteness 
increases and population size decreases, the importance of this enhanced level of GP care also increases, while the likelihood of having locally-available specialist services decreases. ${ }^{1}$ The proportion of rural and remote GPs who are procedurally active, ${ }^{1}$ however, is declining, despite Rural Medical Generalist Programs ${ }^{2}$ designed to bolster the procedural GP workforce.

These circumstances create an imperative for empirical research to inform procedural GP workforce development. Unfortunately, most recent national workforce analyses omit modelling GP procedural activity in separate disciplines because of data limitations including the ability to identify the size and characteristics of the procedural GP workforce. ${ }^{1,3}$

It is known that there is a marked gradient in the proportion of the GP workforce who provide procedural services when measured against the Australian Standard Geographical Classification Remoteness Areas (ASGC-RA) system with remote and very remote communities having the highest proportion of procedural GPs. ${ }^{1,4}$ However, the magnitude and significance of associations between different procedural disciplines and community population size are less well understood. This is despite recent research which reveals that GP workloads are more strongly associated with community population size than with geographical remoteness. ${ }^{5}$ Moreover, little is known about how the workload and on-call patterns of procedural GPs differ from those of non-procedural GPs.

This study investigates patterns of GP procedural activity in anaesthetics, obstetrics, surgery and emergency medicine services. Associations are measured between GP procedural activities and

i. geographical remoteness and population size of practice location;

ii. hours worked in hospitals and in total; and

iii. availability for on-call

\section{Method}

This study uses data from the Medicine in Australia: Balancing Employment and Life (MABEL) national annual longitudinal panel survey of Australian doctors. MABEL's wave 1 (2008) sampling frame was the Australasian Medical Publishing Company's Medical Directory, which comprised 54,746 doctors, of whom 22,137 were GPs. 3,873 GPs responded to wave 1 . Subsequent waves also include small top-up samples of newly registered doctors. Only GPs responding to waves 4, 5 or 6 (in 2011-2013) were included in this analysis. The MABEL surveys are available on the study website (http://mabel.org.au/) and details of the MABEL study protocol, response rates and an analysis of non-response bias have been published elsewhere. ${ }^{6,7}$ In all analyses sampling weights were used to adjust for survey non-response and attrition biases associated with respondents' age, gender, geographic location, jurisdiction, and participation payments. ${ }^{7,8}$ 
From wave 4, GPs were asked "Do you practise in any of anaesthetics, obstetrics, surgery, emergency medicine or none of these", with multiple selections allowed. The probability of remaining procedurally active between consecutive waves (for individual GPs responding to consecutive surveys) was calculated for each group of procedural GPs.

Geographic location of their main place of work was coded according to seven Modified Monash Model (MMM) categories (metropolitan; regional >50000; large rural 15000-50000; medium rural 5000-15000; small rural <5000; remote; very remote) using Australian Bureau of Statistics 2011 census data for Urban Centres Localities. Logistic regression models tested univariate associations between procedural activity and (a) MMM categories (b) Gender and (c) Age group in wave 4. GPs reported their number of hours per week worked, excluding on-call, in each of 8 different settings, and in total. For this study the settings were re-coded to 4 different categories (private rooms, public hospital, private hospital and all other settings). Availability for on-call was a binary variable based on whether GPs did any on-call.

Mean hours worked per setting and the total hours worked were calculated for each group of procedural GPs in waves 4, 5 and 6, and a grand mean calculated across the 3 waves. Multiple linear regression models tested associations between procedural activity and log transformed (a) Hours worked in hospitals and (b) Total hours worked. Multiple logistic regression models tested associations between procedural activity and on-call. Multiple regression models adjusted for age group $(<35 ; 35-<45 ; 45-<55 ; 55-<65 ; \geq 65)$, gender, MMM categories, international medical graduate (IMG) status (yes/no), business relationship (principal/partner; associate; salaried; contracted; other) and practice size (number of GPs in the practice). These models were also assessed to determine whether interactions between age group, gender, MMM categories and IMG status made any substantive difference to estimates and statistical significance of associations with procedural activity.

Calculations were performed using StataSE 13 (StataCorp, College Station, TX, USA). A significance level of $5 \%$ was used.

Ethics approval was from the University of Melbourne Faculty of Economics and Commerce Human Ethics Advisory Group (Ref. 0709559) and from the Monash University Standing Committee on Ethics in Research Involving Humans (Ref. CF07/1102-2007000291).

\section{Results}

3287, 3361 and 2893 clinically active GPs responded to waves 4, 5 and 6 of the MABEL survey. After excluding GP locums and those with missing geographical data, responses were available from 3211, 
3269 and 2821 GPs in waves 4, 5 and 6, respectively. These 9301 survey responses were from 4638 individuals.

185 (4.0\%), 310 (6.7\%), 440 (9.5\%) and 836 (18.0\%) GPs reported undertaking anaesthetics, surgery, obstetrics and emergency medicine, respectively, during any of waves 4, 5 or 6 . (Table 1) Approximately $27 \%$ of procedural GPs provided more than one type of procedural service. Most GP anaesthetists (79\%) provided other types of procedural services, as did approximately half of GP obstetricians and GP surgeons and one-third of emergency GPs.

Analysis of 4663 transitions (pairs of observations) revealed an $86 \%$ probability of remaining a GP anaesthetist between consecutive survey waves with corresponding probabilities amongst GPs providing emergency, obstetric and surgical services $78 \%, 76 \%$ and $53 \%$ respectively.

In wave 4, male GPs were 7.2 times $(95 \% \mathrm{Cl} 4.1,12.8)$ more likely to be GP anaesthetists, 3.6 times $(95 \% \mathrm{Cl} 2.0,6.4)$ more likely to be GP surgeons, 2.2 times $(95 \% \mathrm{Cl} 1.6,3.0)$ more likely to provide emergency services, and no more likely to be GP obstetricians than females. In wave 4 GPs 65 or older were $19.0(95 \% \mathrm{Cl} 4.7,77.4)$ times less likely to practise anaesthetics and $3.1(95 \% \mathrm{Cl} 1.5,6.4)$ and $2.1(95 \% \mathrm{Cl} 1.3,3.2)$ times less likely, respectively, to practise obstetrics and emergency medicine compared to GPs aged under 65.

Significant gradients of increasing odds of GP procedural (non-surgical) activity were identified as rurality increased and location population size decreased with the MMM classification. (Figure 1a-d) This pattern plateaued in medium and smaller sized rural and remote locations for GPs practising anaesthetics and obstetrics.

The mean numbers of hours worked in hospital settings, especially public hospital settings, were higher for procedural GPs than for non-procedural GPs. (Table 2) In general, procedural GPs worked similar hours to non-procedural GPs in their private consultation rooms and in all other settings, but on average worked between 5 (GP Surgeons) and 10 (GP Anaesthetists) more hours in total each week. Regression modelling reveals that procedural GPs work significantly more total hours and more hours in a hospital each week than non-procedural GPs. (Table 3) In wave 4 this equated to GPs practising anaesthetics, obstetrics, surgery, and emergency medicine providing $8 \%(95 \% \mathrm{Cl} 0,16)$, $13 \%(95 \% \mathrm{Cl} 8,19), 8 \%(95 \% \mathrm{Cl} 2,15)$ and $18 \%(95 \% \mathrm{Cl} 13,23)$, more total hours each week, respectively.

Procedural GPs (non-surgical) also had significantly higher - between $1.8(95 \% \mathrm{Cl} 1.3,2.5)$ and 3.7 $(95 \% \mathrm{Cl} 2.7,5.2)$ times - odds of being on-call compared to non-procedural GPs. (Table 4) 
After adjusting for interactions between variables for the models shown in Tables 3 and 4, there were no substantive changes to either the estimates of the main effect of procedural activity or to their statistical significance (results not shown).Discussion

This paper highlights important empirical evidence highly relevant for rural and remote health workforce planning and policy. Strong associations were demonstrated between town population size, geographical remoteness and GP procedural activity: procedurally active GPs are more likely to be located in medium and small rural towns, and in remote and very remote settlements, than in major cities or larger regional centres. These locations are precisely where it has otherwise been difficult to recruit and retain GPs, suggesting that policies which build Australia's procedurally active GP workforce may simultaneously help address the otherwise pervasive issue of rural workforce maldistribution.

Rural workforce policy makers have recently taken important steps towards building Australia's procedural GP workforce, as demonstrated by the increasing support for rural medical generalist training programs ${ }^{9}$ and the funding of continuing professional development for procedural GPs. ${ }^{10}$ Next steps, however, should include strategic decision making about which rural and remote communities to target for the renewed and expanded hospital infrastructure and human resourcing necessary to fully support GP procedural services in these locations and surrounding regions. ${ }^{11}$

Procedural GPs experience greater hospital and total working hours and a higher burden of on-call without any reduction in their private consultation hours compared to non-procedural GPs. Importantly, these heavier workloads are independent of geographical remoteness/population size, so are in addition to the increased workloads experienced by rural and remote GPs compared to their metropolitan counterparts. ${ }^{5}$ Recently qualified GPs choosing rural practice are often interested in developing and maintaining procedural skills, ${ }^{12}$ but they also increasingly seek a satisfactory worklife balance. ${ }^{13}$ Heavier workloads associated with rural procedural practice may deter medical graduates from choosing procedural GP disciplines ${ }^{14}$ and from choosing rural practice while existing procedurally-skilled GPs may be deterred from continuing procedural practice. ${ }^{3}$ Conversely, policies and practices which support reasonable workloads for procedural GPs are likely to encourage recruitment and retention in rural practice and continuation of procedural activity. ${ }^{15}$ These are critical issues that must be addressed if current investments in graduating more procedural GPs via the various jurisdictional rural Generalist Pathways are to produce growth and sustainability of the procedural GP workforce.

This article is protected by copyright. All rights reserved 
A range of possible solutions to the higher on-call demands and longer working hours of procedural GPs require further investigation. For example, models of care in which procedural GPs are more integrated as team members may enable some aspects of the care to be more readily shared, thereby creating opportunities for reducing demands on individual procedural GPs. The development of formal and informal networks of procedural GPs across regions also has potential to provide the necessary critical mass of providers to enable rosters to be developed co-operatively with the potential to reduce on-call and improve work-life balance amongst procedural GPs. ${ }^{16}$ Other models of service delivery, including telehealth-support for after-hours emergencies, may similarly reduce the burden of on-call and workload unpredictability for rural and remote procedural GPs by increasing the scope of emergency practice of lesser-trained cadres of health professionals. ${ }^{17}$ Threats to the viability of procedural practice must also be recognised and countered by policy makers. ${ }^{18}$ Corporatised models of primary care, for example, which attract GPs without the skills necessary for an extended scope of practice into rural communities, may not only diminish opportunities for sharing the on-call burden amongst existing practitioners, but also increase the difficulties associated with recruiting suitably skilled GPs willing to provide on-call services to those communities.

This study has considerable strengths, including consistent findings across multiple waves of GP survey data, its national representation, and the large cohort size. However, it is not without limitations, including self-definition of procedural activity by MABEL survey participants and selfreporting of procedural discipline without verification of qualifications. These limitations may partially explain aberrations observed for GP surgeons, such as the unexpectedly low probability of remaining a GP surgeon between waves. Problems of non-response bias and attrition bias may also affect our data, although these concerns are debatable. ${ }^{20}$ The use of survey weights mitigates this risk, and previous analyses of representativeness indicate that MABEL's panel of participants is without any apparent significant bias. ${ }^{6}$

\section{Conclusion}

This important study exemplifies the value of data analyses which separately identify and quantify associations between different types of procedural GPs and their geographic location, gender, age and workload patterns. The study shows that procedural GPs experience longer working hours and higher on-call demands compared to non-procedural GPs. Addressing these deterrents to procedural General Practice is likely to increase the attractiveness of procedural careers to current and aspiring rural GPs. Robust procedural GP workforce supply may, in turn, strengthen the rural primary care workforce in locations that may otherwise face intractable workforce supply issues. 
References

Health Workforce Australia. Health Workforce 2025 - Medical specialties - Volume 3.

Adelaide: HWA, 2012

https://www.hwa.gov.au/sites/uploads/HW2025 V3 FinalReport20121109.pdf (accessed

October 2015).

Health Workforce Australia. Rural medical generalist and dual trained Physician projects.

Adelaide: HWA, 2014

http://www.hwa.gov.au/sites/default/files/HWA Rural\%20Medical\%20Generalist\%20and\% 20Dual\%20Trained\%20Physician\%20projects.pdf (accessed October 2015).

Health Workforce Australia. The GP proceduralist (maternity services) workforce in rural Victoria: future demand analysis. Final report. Adelaide: HWA, 2014

http://www.hwa.gov.au/sites/uploads/HWA\%20GP\%20Proceduralist\%20workforce\%20in\%2 Orural\%20Vic LR.pdf (accessed June 2015).

Rural Health Workforce Australia. Medical practice in rural and remote Australia: National Minimum Data Set (MDS) report as at 30th November 2013. Melbourne: RHWA, 2014 http://www.rhwa.org.au/client images/1741064.pdf (accessed October 2015).

McGrail MR, Humphreys JS, Joyce CM, Scott A, Kalb G. How do rural GPs' workloads and work activities differ with community size compared with metropolitan practice? Aust J Prim Health 2011; 18: 228-233.

Joyce CM, Scott A, Jeon S-H, et al. The "Medicine in Australia: Balancing Employment and Life (MABEL)" longitudinal survey - Protocol and baseline data for a prospective cohort study of Australian doctors' workforce participation. BMC Health Serv Res 2010; 10: 50.

7 Yan W, Cheng TC, Scott A, et al. Medicine in Australia: Balancing Employment and Life (MABEL). Australian Economic Review 2011; 44: 102-112. Institute of Applied Economics and Social Research, 2015 
http://mabel.org.au/ data/assets/pdf file/0006/1389525/MABEL User Manual Wave 6. pdf (accessed July 2015).

9 Australian College of Rural \& Remote Medicine. Rural Generalist Medicine Brisbane: ACRRM; 2014. http://www.acrrm.org.au/the-college-at-work/rural-generalist-medicine (accessed May 2015.

10 Australian College of Rural \& Remote Medicine. Rural Procedural Grants Program (RPGP) Brisbane: ACRRM; 2015. http://www.acrrm.org.au/continuing-development/assistanceand-grants/rpgp (accessed September 2015.

11 Rural Doctors Association of Victoria. RDAV Rural emergency services position paper 2009. Mount Beauty: 2009 http://www.rdav.com.au/Uploads/Documents/2009RDAVPosStatemEmServs 20140811110 749.pdf (accessed September 2015).

12 Laurence CO, Williamson V, Sumner KE, Fleming J. "Latte rural": the tangible and intangible factors important in the choice of a rural practice by recent GP graduates. Rural Remote Health 2010; 10: 1316.

13 Shrestha D, Joyce CM. Aspects of work-life balance of Australian general practitioners: determinants and possible consequences. Aust J Prim Health 2011; 17: 40-47.

14 Tolhurst HM, Stewart SM. Balancing work, family and other lifestyle aspects: a qualitative study of Australian medical students' attitudes. Med J Aust 2004; 181: 361-364.

15 Glazebrook RM, Harrison SL. Obstacles and solutions to maintenance of advanced procedural skills for rural and remote medical practitioners in Australia. Rural Remote Health 2006; 6: 502.

16 Campbell AM, Brown J, Simon DR, Young S, Kinsman L. Leading the rebirth of the rural obstetrician. Med J Aust 2014; 201: 667-670.

17 WA Country Health Service. Emergency Telehealth Service Perth: WACHS; 2011. http://www.wacountry.health.wa.gov.au/index.php?id=778 (accessed September 2015.

18 Rural Doctors Association of Australia, Monash University School of Rural Health. Viable models of rural and remote practice: Stage 1 and Stage 2 report. Canberra: RDAA, 2003 
http://www.rdaa.com.au/Uploads/Documents/Stage\%201\%20and\%20Stage\%202\%20Repor ts 20110224092844.pdf (accessed October 2015).

19 Rural Doctors Association of Victoria. The Victorian rural and regional workforce pipeline forum: hard questions. Mount Beauty: 2013

http://www.rdav.com.au/Uploads/Documents/2013\%20PipelineForumReport 2015012301 2450.pdf (accessed October 2015).

20 Cheng TC, Trivedi PK. Attrition bias in panel data: A sheep in wolf's clothing? A case study based on the MABEL survey. Health Econ 2015; 24: 1101-1117.

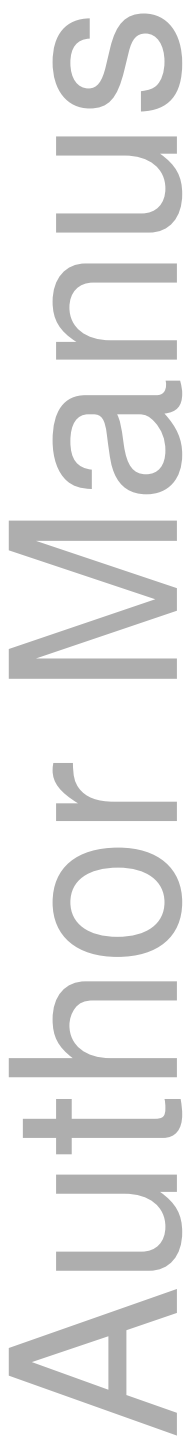

This article is protected by copyright. All rights reserved 


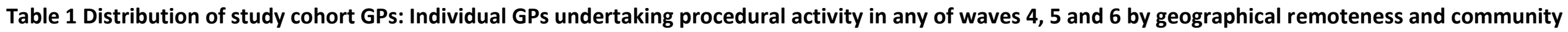
population size in wave of first response

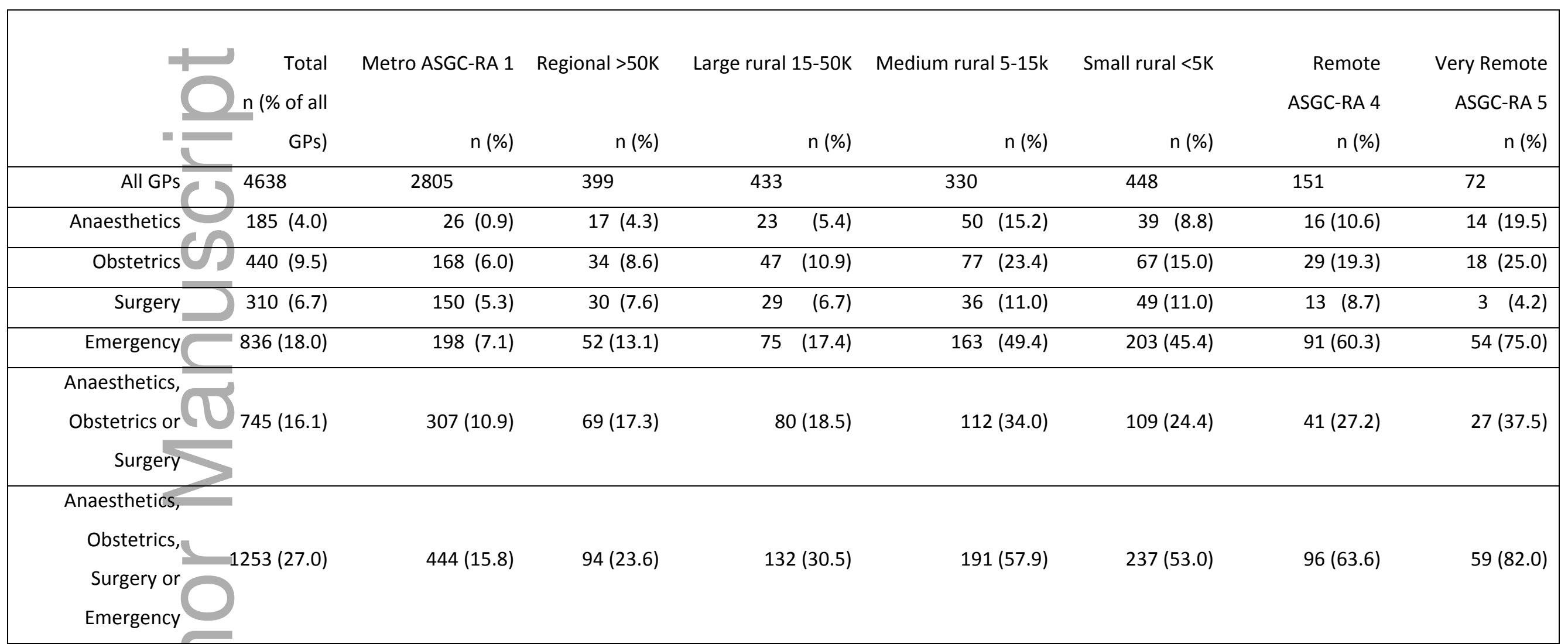

ASGC-RA Australian Standard Geographical Classification - Remoteness Areas

$\mathrm{K}=1000$

Table 2 Mean hours worked by GPs in a usual week in different work settings in waves 4,5 and 6, by procedural activity

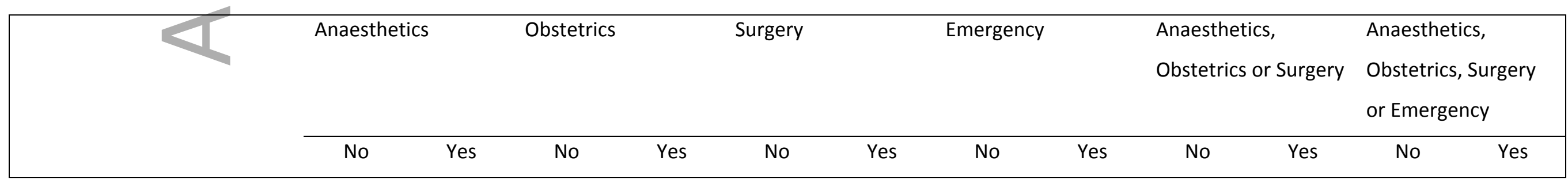

This article is protected by copyright. All rights reserved 


\begin{tabular}{|c|c|c|c|c|c|c|c|c|c|c|c|c|}
\hline Private consultation rooms & 31.4 & 27.5 & 31.3 & 31.3 & 31.1 & 34.4 & 31.4 & 30.2 & 31.3 & 31.2 & 31.5 & 30.4 \\
\hline Public hospital & 1.3 & 13.4 & 1.3 & 7.4 & 1.6 & 3.0 & 0.7 & 9.0 & 1.2 & 5.9 & 0.5 & 6.8 \\
\hline Private hospital & 0.6 & 2.6 & 0.6 & 0.6 & 0.5 & 3.4 & 0.6 & 0.8 & 0.4 & 2.3 & 0.4 & 1.8 \\
\hline All other settings & 4.8 & 5.8 & 4.8 & 5.3 & 5.0 & 3.1 & 4.6 & 6.8 & 4.9 & 4.5 & 4.7 & 5.6 \\
\hline TOTAL HOURS WORKED & 38.1 & 48.5 & 38.0 & 44.6 & 38.1 & 43.5 & 37.3 & 46.5 & 37.7 & 43.6 & 37.0 & 44.3 \\
\hline
\end{tabular}

Table 3 Multiple linear regression model estimates of (a)hours worked in a hospital setting and (b)total hours worked each week, by procedural activity after adjustments $^{*}$

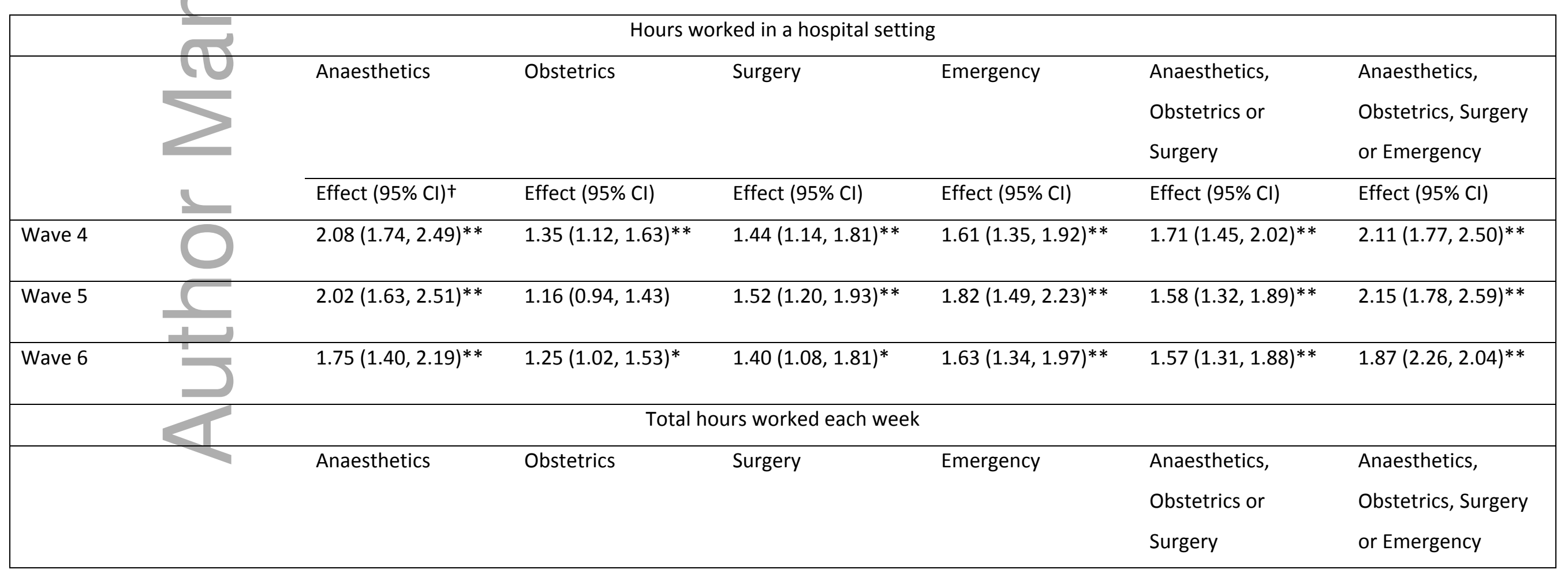

This article is protected by copyright. All rights reserved 


\begin{tabular}{|lllllll}
\hline & Effect $(95 \% \mathrm{Cl})$ & Effect $(95 \% \mathrm{Cl})$ & Effect $(95 \% \mathrm{Cl})$ & Effect $(95 \% \mathrm{Cl})$ & Effect $(95 \% \mathrm{Cl})$ \\
\hline Wave 4 & $1.08(1.00,1.16)^{*}$ & $1.13\left(1.08,1.19^{* *}\right.$ & $1.08(1.02,1.15)^{* *}$ & $1.18(1.13,1.23)^{* *}$ & $1.10(1.06,1.15)^{* *}$ & $1.15(1.11,1.19)^{* *}$ \\
\hline Wave 5 & & & & & \\
& & $1.10(1.03,1.17)^{* *}$ & $1.09(1.04,1.14)^{* *}$ & $1.07(1.01,1.13)^{*}$ & $1.18(1.13,1.23)^{* *}$ & $1.08(1.04,1.12)^{* *}$ \\
\hline Wave 6 & & & & & $1.13(1.09,1.17)^{* *}$ \\
\hline
\end{tabular}

* $\quad \mathrm{p}<0.05$

** $p<0.01$

Reference categories are non-proceduralists (eg. GP anaesthetists compared with non-GP anaesthetists)

tEffect is the exponentiated regression coefficient, and the values can be interpreted as the percentage increase in hours worked (eg. 1.28 represents a $28 \%$ increase in hours worked compared to the reference group)

${ }^{\ddagger}$ Adjustments are for age, gender, geographical remoteness and community population size (Modified Monash Model categories), international medical graduate status, business relationship and practice size

Table 4 Multiple logistic regression model estimates of participation (or not) in an on-call roster by GP procedural activity, after adjustments ${ }^{\dagger}$

\begin{tabular}{|c|c|c|c|c|c|c|c|}
\hline & 2 & Anaesthetics & Obstetrics & Surgery & Emergency & $\begin{array}{l}\text { Anaesthetics, } \\
\text { Obstetrics or } \\
\text { Surgery }\end{array}$ & $\begin{array}{l}\text { Anaesthetics, } \\
\text { Obstetrics, Surgery } \\
\text { or Emergency }\end{array}$ \\
\hline & & Odds Ratio $(95 \% \mathrm{Cl})$ & Odds Ratio $(95 \% \mathrm{Cl})$ & Odds Ratio $(95 \% \mathrm{Cl})$ & Odds Ratio $(95 \% \mathrm{Cl})$ & Odds Ratio $(95 \% \mathrm{Cl})$ & Odds Ratio $(95 \% \mathrm{Cl})$ \\
\hline Wave 4 & 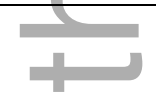 & $2.0(1.1,3.6)^{*}$ & $2.4(1.7,3.3)^{* *}$ & $1.3(0.8,2.0)$ & $3.7(2.7,5.2)^{* *}$ & $1.8(1.4,2.3)^{* *}$ & $2.4(1.9,3.1)^{* *}$ \\
\hline Wave 5 & & $2.6(1.5,4.4)^{* *}$ & $1.8(1.3,2.5)^{* *}$ & $1.0(0.7,1.5)$ & $2.5(1.9,3.3)^{* *}$ & $1.4(1.1,1.9)^{* *}$ & $1.9(1.5,2.4)^{* *}$ \\
\hline Wave 6 & & $3.3(1.5,7.0)^{* *}$ & $2.0(1.4,2.9)^{* *}$ & $1.2(0.7,2.0)$ & $3.4(2.4,4.7)^{* *}$ & $1.7(1.2,2.3)^{* *}$ & $2.2(1.7,2.9)^{* *}$ \\
\hline
\end{tabular}

* $\quad p<0.05$

** $p<0.01$

This article is protected by copyright. All rights reserved 
Reference categories are non-procedural GPs (eg. GP anaesthetists compared with non-GP anaesthetists)

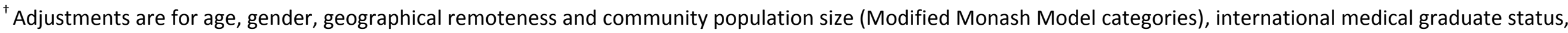
business relationship and practice size

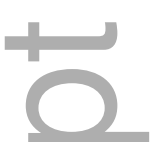

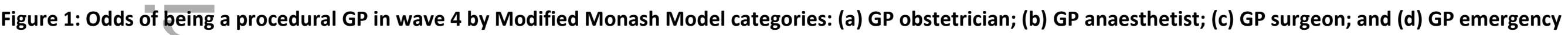
services

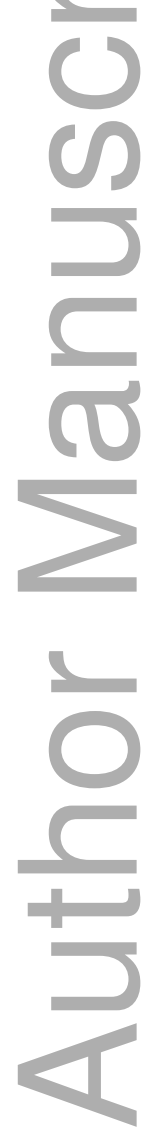

This article is protected by copyright. All rights reserved 


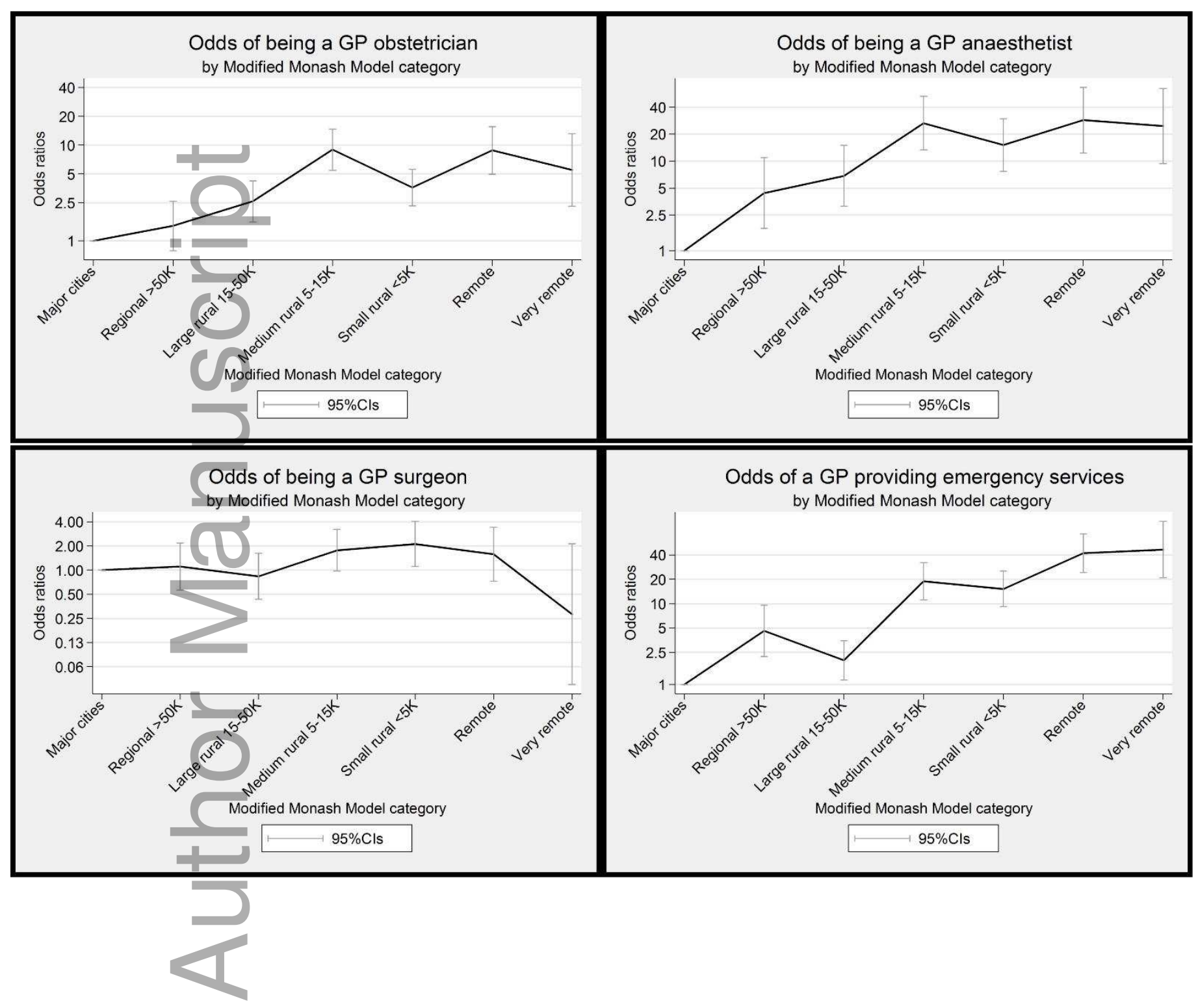

This article is protected by copyright. All rights reserved 


\section{University Library}

\section{- M M I N E R VA A gateway to Melbourne's research publications}

Minerva Access is the Institutional Repository of The University of Melbourne

Author/s:

Russell, DJ;McGrail, MR

Title:

How does the workload and work activities of procedural GPs compare to non-procedural GPs?

Date:

2017-08

Citation:

Russell, D. J. \& McGrail, M. R. (2017). How does the workload and work activities of procedural GPs compare to non-procedural GPs?. Aust J Rural Health, 25 (4), pp.219-226. https://doi.org/10.1111/ajr.12321.

Persistent Link:

http://hdl.handle.net/11343/291702 